\title{
Criminologie
}

\section{Les liens entre jeux de hasard et d'argent, activités policières et demandes d'aide}

\author{
Marc Alain, Danny Dessureault et Natacha Brunelle
}

Volume 45, numéro 2, automne 2012

Crime et jeux de hasard

URI : https://id.erudit.org/iderudit/1013719ar

DOI : https://doi.org/10.7202/1013719ar

Aller au sommaire du numéro

\section{Éditeur(s)}

Les Presses de l’Université de Montréal

ISSN

0316-0041 (imprimé)

1492-1367 (numérique)

Découvrir la revue

Citer cet article

Alain, M., Dessureault, D. \& Brunelle, N. (2012). Les liens entre jeux de hasard et d'argent, activités policières et demandes d'aide. Criminologie, 45(2), 27-49.

https://doi.org/10.7202/1013719ar
Résumé de l'article

Cet article présente les résultats d'une démarche de suivi des effets macrosociologiques liés à l'implantation d'une toute nouvelle offre de jeux de hasard et d'argent présentée par Loto-Québec dans un contexte péri-urbain : le Salon de jeu de Trois-Rivières, un lieu de loisirs qui regroupe sous un même toit des appareils de loterie vidéo, des tables de poker et une roulette virtuelle. Plusieurs collectes de données ont servi à matérialiser ce bilan des effets, soit, d'une part, un suivi chronologique des appels logés au service de police de la ville et, d'autre part, un suivi des demandes d'aide et de référence pour problème de jeu pathologique, demandes tenues à jour par l'organisme Domrémy MCQ. L'analyse des données policières se révèle fidèle à ce que la littérature permet de penser, soit qu'il n'y a pas de lien entre l'introduction d'une nouvelle offre légalisée de jeux de hasard et d'argent et la criminalité. En revanche, l'absence de liens entre cette nouvelle offre et le nombre de demandes d'aide, quant à elle, va dans le sens contraire de ce que la littérature a établi. Nous proposons que la principale raison expliquant ces phénomènes tient dans le peu de succès qu'a connu le Salon de jeu pendant la période où nos travaux se sont déroulés. 


\title{
Les liens entre jeux de hasard et d'argent, activités policières et demandes d'aide
}

\author{
Marc Alain ${ }^{1}$ \\ Professeur \\ Département de psychoéducation, Université du Québec à Trois-Rivières \\ marc.alain@uqtr.ca \\ Danny Dessureault \\ Professeur \\ Département de psychoéducation, Université du Québec à Trois-Rivières \\ danny.dessureault@uqtr.ca \\ Natacha Brunelle \\ Professeure titulaire \\ Département de psychoéducation, Université du Québec à Trois-Rivières \\ Natacha.Brunelle@uqtr.ca
}

RÉSUMÉ - Cet article présente les résultats d'une démarche de suivi des effets macrosociologiques liés à l'implantation d'une toute nouvelle offre de jeux de hasard et d'argent présentée par Loto-Québec dans un contexte péri-urbain: le Salon de jeu de Trois-Rivières, un lieu de loisirs qui regroupe sous un même toit des appareils de loterie vidéo, des tables de poker et une roulette virtuelle. Plusieurs collectes de données ont servi à matérialiser ce bilan des effets, soit, d'une part, un suivi chronologique des appels logés au service de police de la ville et, d'autre part, un suivi des demandes d'aide et de référence pour problème de jeu pathologique, demandes tenues à jour par l'organisme Domrémy MCQ. L'analyse des données policières se révèle fidèle à ce que la littérature permet de penser, soit qu'il n'y a pas de lien entre l'introduction d'une nouvelle offre légalisée de jeux de hasard et d'argent et la criminalité. En revanche, l'absence de liens entre cette nouvelle offre et le nombre de demandes d'aide, quant à elle, va dans le sens contraire de ce que la littérature a établi. Nous proposons que la principale raison expliquant ces phénomènes tient dans le peu de succès qu'a connu le Salon de jeu pendant la période où nos travaux se sont déroulés.

1. Toute correspondance concernant cet article doit être adressée à monsieur Marc Alain.

Criminologie, vol. 45, n 2 (2012) 
MOTS-CLÉS - Jeux de hasard et d'argent, criminalité induite, activité policière induite, casinos légaux, jeu pathologique, santé publique.

\section{Introduction $^{2}$}

Larticle que nous présentons ici fait état, dans une perspective empirique et descriptive, des résultats obtenus au terme de trois années de suivi de l'implantation du Salon de jeu de Trois-Rivières. En tant que tel, cet article doit être compris à la hauteur de son principal objectif, soit de décrire aussi précisément que possible ce que les indices macrosociologiques mesurés permettent de révéler de la controverse importante qui a présidé à l'ouverture du Salon de jeu de Trois-Rivières. Nous commencerons donc par faire état de cette controverse et de ses origines; par la suite, une courte revue de littérature sur la question des liens entre des indicateurs macrosociologiques larges - dont les fluctuations de la criminalité - et l'existence de modalités de jeux de hasard et d'argent similaires à ce qu'offre le Salon de jeu sera présentée. Nous ferons ensuite état des résultats empiriques de notre démarche de recherche. Trois sources de données seront ici mises à contribution afin d'établir les impacts liés à l'ouverture du Salon de jeu, en partant des premières mesures effectuées quelques mois avant l'ouverture du salon de jeu en 2007, jusqu'en décembre 2009. Ces trois sources sont: (1) les données d'activités policières, avec ou sans ouverture d'un dossier criminel, telles qu'enregistrées dans le système de gestion des appels à la police de la ville pour les secteurs de patrouille où est situé le Salon de jeu et dans les secteurs environnants de la ville; (2) les données colligées par le centre de réadaptation en dépendance régional Domrémy Mauricie-Centre-du-Québec pour les demandes d'aide et de référence pour les problèmes de jeu pathologique, demandes recensées pour l'ensemble des onze points de service de la région; (3) ces deux premières sources seront appuyées par les données plus qualitatives issues de séances de groupes de discussion qui ont été tenues avec des policiers, gestionnaires, patrouilleurs et enquêteurs, d'une part, et avec des intervenants de la santé dans la région, issus de la santé publique et d'organismes communautaires, d'autre part. Ces séances ont eu lieu à deux reprises pendant les travaux de recherche, et ce, pour les deux groupes

2. Cette recherche a été financée par le Fonds québécois de la recherche sur la société et la culture, programme des Actions concertées. Les analyses, points de vue et conclusions présentés dans cet article n'engagent toutefois que les auteurs. 
et avaient comme principaux objectifs (a) de valider les analyses des tendances observées dans les séries chronologiques et (b) de vérifier si d'autres phénomènes potentiels et difficiles à mesurer par le biais d'indicateurs quantitatifs pouvaient être présents ou non.

\section{Contexte sociopolitique et état de la littérature}

La Société Loto-Québec était, au moment de sa fondation en 1969, la toute première société de loteries d'État au Canada et, à ce moment, la troisième du genre en importance en Amérique du Nord (Loto-Québec, 2007). Elle n'a, depuis, cessé de prendre de l'ampleur en occupant des créneaux plus diversifiés: les loteries sous toutes les formes possibles bien sûr, mais peu à peu aussi, les bingos, les casinos, jusqu'à accaparer, finalement, le créneau des appareils de loterie vidéo (ALV). C'est ce dernier secteur d'activités qui, à plus d'un titre, a suscité les critiques les plus vives à l'endroit des stratégies commerciales de la société d'État. Deux éléments, l'un majeur et l'autre mineur, ont, entre autres, contribué à ces critiques. Le premier tournait autour du phénomène de mieux en mieux documenté de l'accroissement du jeu pathologique et des troubles associés à la dépendance aux ALV. Le second élément, quant à lui, consistait en ceci que ces mêmes ALV grugeaient une part non négligeable des profits engendrés par un autre secteur du jeu légalisé, celui des courses de chevaux. On assistait, pour ce qui est du premier élément, au dévoilement en rafale et à la médiatisation d'une série d'études sur les problèmes de jeu pathologique associés à un accroissement de l'accès aux ALV (Chevalier et al., 2004; Chevalier et Papineau, 2007; Leblond, 2007), un phénomène que plusieurs commentateurs attribuaient à la gourmandise de la société de loterie et à celle de son seul actionnaire, l'État québécois. Et, dans le second cas, c'est en prétextant que le déclin relatif de leur secteur résultait de ce même accroissement de l'accès aux ALV que les administrateurs et promoteurs de l'industrie des courses de chevaux réclamaient des aménagements susceptibles de leur permettre de toucher une part des profits engendrés par les ALV. Loto-Québec dévoilait alors (Loto-Québec, 2007) une stratégie de redéploiement des ALV susceptible d'atténuer ces deux phénomènes: l'implantation et l'ouverture de quatre salons de jeu au Québec, soit dans la couronne nord de Montréal, à Québec, à Mont-Tremblant et à Trois-Rivières. L'implantation de ces salons de jeu, situés dans les cas de Québec et de Trois-Rivières au sein même des hippodromes, était 
alors présentée comme la solution devant permettre d'atténuer en une seule action ces deux problèmes: permettre, d'une part, une réallocation des profits engendrés par les ALV sous forme de dividendes aux gens des courses de chevaux et permettre, d'autre part, un niveau de contrôle plus serré des problèmes de jeu pathologique ${ }^{3}$. C'est ainsi qu'en octobre 2007 s'ouvrait le Salon de jeu de Trois-Rivières, avec, en toile de fond, un débat entre deux positions opposées, soit celle des promoteurs qui minimisaient les problèmes sociaux liés aux jeux de hasard et d'argent et celle des intervenants de la santé qui considéraient qu'une offre supplémentaire de jeux allait s'accompagner d'une hausse des problématiques sociales attachées au phénomène. C'est cette opposition et le fait que les autorités publiques québécoises - essentiellement les ministères de la Sécurité publique et de la Santé et des services sociaux - étaient intéressées à départager les points de vue qui ont en quelque sorte servi de justification à nos travaux empiriques.

Dans le cas plus précis de la question des liens entre la criminalité enregistrée auprès des organismes chargés de son contrôle et les jeux de hasard et d'argent, elle peut être comprise selon au moins deux perspectives. Dans le cas de la première perspective, on réfèrera à ces liens dans une logique plus individuelle et microsociologique, tandis que la seconde est d'ordre plus macrosociologique. Il s'agit de l'exploration des liens potentiels entre une hausse ou une modification de l'offre des jeux de hasard et d'argent, d'une part, et des fluctuations des activités criminelles opportunistes et des problèmes de jeu pathologique, d'autre part. C'est uniquement cette deuxième perspective qui sera exploitée ici. Dans ce cas-ci, les activités criminelles en question sont tout d'abord celles auxquelles auraient recours des joueurs pathologiques ayant épuisé leurs moyens financiers réguliers et légaux (fraudes, contrefaçon de toute sorte, vols par effraction, etc.), mais aussi des activités criminelles plus périphériques, des activités que l'on associe généralement à des organisations plus ou moins structurées: prêts usuraires, racket,

3. Ces modalités prenaient plusieurs formes, dont entre autres: (1) le regroupement des ALV dans un lieu unique et dont l'atmosphère serait très différente de ce que l'on peut trouver dans les petits bars de quartier où l'on trouve plus généralement les ALV; (2) le fait, pour les joueurs, de devoir recourir à une carte prépayée au montant fixe et devant être réapprovisionnée par le joueur à un comptoir central; (3) la présence, sur les lieux, dans les cas de Trois-Rivières et de Québec à tout le moins, «d'ambassadeurs de jeu», des intervenants sociaux dédiés, employés par les organismes locaux de santé publique et chargés de faire la promotion du jeu responsable et des saines habitudes de jeu. 
menaces, intimidation et violences liées aux difficultés éprouvées par les clients des prêteurs à rembourser des sommes dues, etc.

Pourtant, l'établissement de liens macrosociologiques entre le jeu et la criminalité part d'un postulat logique qui émane de constats qui sont, à la base, beaucoup plus microsociologiques. Piscitelli et Albanese (2000), par exemple, évoquent qu'en vertu de la théorie des activités routinières (Cohen et Felson, 1979; Gottfredson et Hirschi, 1991; Felson, 1998), le jeu pourrait être compris comme l'une des facettes de la vie d'individus centrés autour de la recherche de plaisirs immédiats; en ce sens, l'association entre le jeu et la délinquance n'est pas nécessairement directe, elle est plutôt associative ${ }^{4}$. C'est d'ailleurs un peu dans ce sens que Grinols (2000) présente l'argument: comme la plupart des études réalisées auprès de joueurs pathologiques indiquent qu'une proportion significative de ces joueurs reconnaît commettre ou avoir commis des infractions criminelles (Grinols signale une proportion de $62 \%$; Smith et al. (2003), de leur côté, indiquent plutôt une proportion de $50 \%$ ), à partir du moment où l'on étend l'offre de jeu, on devrait normalement constater une hausse de la criminalité.

Or, si cette extension de résultats microsociologiques vers des conséquences d'ordre plus macrosociologique peut paraître logique, dans les faits les travaux qui ne s'en tiennent justement qu'à cette dernière perspective ne parviennent pas à établir de relations solides. C'est sans contredit la question de l'ouverture tous azimuts de casinos un peu partout sur le territoire américain qui a suscité le plus de recherches sur la question des liens jeu-crimes. Powel (2004), par exemple, ainsi que Wilson (2001) se sont spécifiquement intéressés aux impacts des «riverboat casinos », ces anciens bateaux à roue à aubes des fleuves et rivières des États du Sud américain transformés en casinos flottants plus ou moins itinérants. Si, selon les analyses de Powel, la présence des «riverboat casinos» n'a eu aucun impact mesurable sur les indices de qualité de vie utilisés, l'auteure montre que les taux de criminalité en général et les taux de crime contre la propriété ont, de leur coté et sans qu'elle

4. L'exploitation de la théorie des activités routinières pour expliquer l'association entre jeu et criminalité ne semble pas faire l'unanimité; en effet, Slutske, Blaszczynski et Martin (2009), de même que Ladouceur, Blaszczynski et Pelletier (2004), montrent que si certains joueurs pathologiques, généralement des jeunes hommes, correspondent d'assez près à ce que la théorie prétend, en revanche, la grande majorité des joueurs pathologiques développe cette habitude comme une activité susceptible de combler un mal de vivre aux résonances plus profondes et ancrées dans la personnalité même du joueur. On parle ici, notamment, des femmes et des hommes de tranches d'âge plus élevées. 
n'en donne précisément la raison, significativement baissé. Wilson (2001), quant à lui, et à la suite de la multiplication de ces «riverboat casinos» en Indiana, ne détecte aucun des indices de fluctuation de la criminalité attendus par la théorie des activités routinières.

D'autres auteurs (Chang, 1996; Mun, 2003 ; Wilbon, 2005), reconnaissant d'emblée l'absence de liens vérifiables entre les taux de criminalité et l'ouverture des casinos, en viennent cependant à évoquer le fait que la plupart des cas répertoriés le sont dans des contextes de modalités de contrôle étatique assez serrées; l'idée devient donc tentante de vérifier l'état de ces liens entre les fluctuations de la criminalité et la présence de casinos dans des contextes où de tels contrôles sont moins présents. Ici, on fait notamment référence à la présence des casinos dans des territoires autochtones; là aussi, cependant, et Mays, Casillas et Maupin (2007) le montrent clairement, le lien entre les deux phénomènes ne se vérifie pas plus. Au Canada également, et tant dans des territoires autochtones qu'urbains, l'existence d'un lien entre la présence des casinos et des modulations de la criminalité ne se vérifie pas non plus (Piscitelli et Albanese, 2000; Smith et al., 2003).

Compte tenu des grandes conclusions de ces travaux, nous ne devrions vraisemblablement pas nous attendre à des fluctuations significatives de la criminalité et des activités des agences pénales dans la région de Trois-Rivières après l'ouverture de son Salon de jeu. Mais outre la crainte des impacts potentiels de l'ouverture du Salon de jeu sur la criminalité et l'activité policière, celle des impacts sur la santé publique a probablement encore plus défrayé les manchettes. Cette question des impacts de l'introduction d'une nouvelle offre légale de jeux de hasard et d'argent sur les indicateurs de santé publique fait appel, quant à elle, à un autre pan de recherche qu'il convient ici de survoler.

Korn et Shaffer (1999; voir aussi Korn, 2000; Korn et Skinner, 2000; Shaffer et Korn, 2002) vont être à l'origine de l'idée de comprendre la question des jeux de hasard et d'argent à partir de l'optique de la santé publique. Ce faisant, Korn et Shaffer (1999) et, un peu plus tard, Korn, Gibbins et Azmier (2003), examinent les retombées de la présence du jeu légalisé sous l'angle de perspectives que l'on retrouve plus généralement associées aux maladies et à leur prévention. Shaffer, Labrie et Laplante (2004) vont à ce titre pousser encore plus loin l'analogie en proposant d'examiner le jeu légalisé comme une toxine de santé publique et, donc, de voir comment les autorités publiques peuvent interve- 
nir de manière à réduire l'exposition des populations fragiles à cette «toxine». Tout à fait comme on pourrait comprendre les avantages et inconvénients d'ouvrir une usine de produits potentiellement dangereux près d'un quartier résidentiel (c.-à-d. les avantages économiques larges versus les dangers d'une exposition accidentelle à des produits chimiques nocifs), on évoque dans le cas des jeux légalisés une série d'éléments d'appui à des initiatives susceptibles de faire augmenter l'offre, de même qu'une série d'éléments critiques susceptibles de la freiner. Whynne et Shaffer (2003) proposent, pour ce qui est des avantages, et outre la question d'une hausse des emplois, des revenus et des taxes potentiellement apportés par l'augmentation de l'offre de jeu légalisé, que le développement du jeu légalisé permettrait une attrition des modalités illégales de jeu et, en corollaire, de la criminalité régnant autour de ces modalités. Mais c'est sur le plan de la santé publique que les désavantages semblent être les plus marqués. Whynne et Shaffer (2003), de même que Afifi, Cox, Martens, Sareen et Enns (2010) et Pearce, Mason, Hiscock et Day (2008) avancent que l'augmentation de l'offre de jeu légalisé entraîne deux conséquences majeures sur le plan de la santé publique, soit, d'une part, l'augmentation du nombre de personnes aux prises avec un problème de dépendance au jeu et, d'autre part, les retombées négatives de ce problème chez les proches du joueur ou de la joueuse (voir particulièrement Afifi et al. [2010] à cet égard).

Ce sont donc deux grandes questions, elles-mêmes issues des constats de la recherche dans le domaine des impacts liés à l'ouverture des casinos, qui serviront de toile de fond à la présentation de nos résultats empiriques. Serons-nous en mesure, en premier lieu, de détecter des fluctuations de la criminalité, ou à tout le moins de l'activité policière autour et dans le secteur du Salon de jeu de Trois-Rivières et qu'en sera-t-il, d'autre part, des fluctuations d'au moins un indicateur de santé publique, soit le nombre de demandes d'aide et de référence pour problèmes de jeu pathologique. Pour ce qui est de la première question, et si l'on se fie aux connaissances empiriques dont nous avons fait le survol, nous ne devrions pas observer comme tel de modifications significatives. Comme, cependant, ces préoccupations de l'augmentation des indices de criminalité et d'activités policières générées par cette nouvelle offre de jeu légalisé ont été au cœur des débats qui ont entouré l'ouverture du Salon de jeu à Trois-Rivières, il conviendra d'en faire l'état. Quant au second phénomène, celui des impacts de cette nouvelle offre de jeu sur l'indicateur de santé publique dont nous disposons, 
mentionnons que la société Loto-Québec déclare depuis plusieurs années maintenant promouvoir une offre de jeu responsable et qu'à ce titre, toutes les précautions d'usage seraient prises au Salon de jeu pour éviter le développement d'habitudes pathologiques chez la clientèle ${ }^{5}$. Nous pourrons donc observer jusqu'à quel point les stratégies de prévention mises en place au Salon de jeu tant par Loto-Québec que par les agences locales de santé publique vont se refléter ou non dans la fluctuation des demandes d'aide.

\section{Aspects méthodologiques}

\section{Criminalité et activités policières}

Nous l'avons vu plus haut, il ne semble pas exister de liens démontrés entre l'ouverture d'un casino et des fluctuations dans l'activité criminelle enregistrée. À ce titre, on peut penser que les larcins et fraudes éventuellement commis par des joueurs incapables de ne pas succomber à leur habitude sont assez peu susceptibles d'être classifiés comme des crimes graves, des délits, donc, qui vont aussi être moins susceptibles de se retrouver dans les «chiffres» de la police.

De plus, et bien qu'encore une fois une telle démonstration ne semble pas avoir été faite, certaines des formes de criminalité opportuniste qui pourraient être occasionnées par l'ouverture d'un casino - on peut penser au prêt usuraire par exemple - sont généralement du ressort de la criminalité organisée et donc, également peu susceptibles de transpirer de ces mêmes chiffres ${ }^{6}$. Ce sont pour ces raisons que nous ne recourons pas uniquement à une étude de la chronologie des délits rapportés au Service de la sécurité publique de la Ville de Trois-Rivières (SSPTR) et que nous avons tenu deux entretiens en groupe de discussion avec des enquêteurs, répartiteurs de patrouille et directeurs de service du SSPTR, le premier approximativement un an après l'ouverture du Salon de jeu et le second, deux ans après cette même ouverture. Ces entretiens nous permettront, entre autres, de tirer au clair la ques-

5. Blaszczynski, Ladouceur et Shaffer (2004) ont, à partir du modèle dit de Reno, proposé un cadre scientifiquement éprouvé de jeu responsable. C'est la mise en place de modalités efficaces de prévention d'habitudes pathologiques de jeu qui vient en premier lieu des considérations qui visent le jeu responsable.

6. Et ce, sans compter bien sûr qu'il s'agit ici d'un cas type de crime «sans victime», si tant est que ni le créancier ni le débiteur n'auraient tendance à porter plainte officiellement en cas de non-respect, de part ou d'autre, des clauses du «contrat». 
tion des fluctuations potentielles dans l'activité des membres locaux du crime organisé, activités qui ne transparaissent pas vraiment dans les statistiques de la criminalité, mais dont la plupart des policiers de terrain ont vent. De plus, nous séparerons, d'entre les données policières, celles qui sont liées à l'ouverture d'un dossier criminel et celles qui ne le sont pas. En effet, l'analyse des activités policières sans ouverture de dossier permet de vérifier si l'ouverture du Salon de jeu pourrait ou non être accompagnée par des incivilités ou des incidents, des plaintes des citoyens qui vivent à proximité de l'endroit et autres petits désordres qui ne nécessitent pas comme tel de procéder à l'ouverture d'un dossier criminel. Les chiffres analysés sont ceux des secteurs de patrouille situés tout autour du Salon de jeu, soit des quartiers de la ville relativement défavorisés ${ }^{7}$.

\section{Les demandes d'aide et de référence pour problèmes} de jeu pathologique

Le centre de réadaptation en dépendance Domrémy Mauricie-Centredu-Québec demeure la principale ressource d'accueil de deuxième ligne pour les personnes souffrant d'un problème de dépendance et pour leurs proches. C'est également cet organisme qui compile l'ensemble des demandes adressées à l'un ou l'autre des 11 centres de services que l'on trouve dans la région. Ce sont ces chiffres qui nous ont été communiqués et qui feront l'objet d'analyses de séries chronologiques sur le modèle de celles des données policières. Tout comme dans le cas des données policières, les analyses quantitatives seront appuyées par les données qualitatives issues de la tenue de groupes de discussion. L'exercice, réalisé à deux reprises pendant la durée des travaux de recherche, a réuni des représentants du milieu de la santé, qu'il s'agisse de représentants d'organismes publics, parapublics ou communautaires. Dans ce cas, les propos des intervenants devraient nous permettre de vérifier jusqu'à quel point des phénomènes impossibles à déceler dans le nom-

7. Notons que nous avons exclu de nos analyses l'ensemble des appels logés directement au quartier général, précisément situé dans ces mêmes secteurs: d'une part, le volume d'appels est très important (plus du double de l'ensemble des données recueillies dans les secteurs voisins) tandis que, d'autre part, il n'a absolument pas fluctué tout au long des trois ans de collecte des données. Les appels enregistrés au quartier général correspondent tout d'abord à l'ensemble des appels pour lesquels aucune donnée de localisation géographique (adresse et code postal) n'existe et, ensuite, aux activités qui ont lieu en tant que telles à cet endroit (essentiellement des demandes d'information de toutes sortes). 
bre des demandes d'aide ne joueraient pas, des phénomènes tels qu'un déplacement des habitudes, ou des lieux où la demande d'aide est adressée.

\title{
Les résultats de trois années de suivi
}

\author{
Criminalité et activités policières dans les quartiers \\ limitrophes du Salon de jeu
}

Comme en font foi les deux tableaux suivants, les années 2006-2007, 2007-2008 et 2008-2009 fluctuent de manière aléatoire d'un mois à l'autre sans que l'on ne décèle quelque tendance particulière ${ }^{8}$.

TABLEAU 1

Statistiques descriptives des appels logés par mois à la police dans les secteurs îlots et atomes où est situé le Salon de jeu et dans les secteurs de patrouille environnants pour les trois derniers mois de 2006 et pour les années 2007, 2008 et 2009

\begin{tabular}{|l|c|c|c|c|c|c|c|}
\hline & & \multicolumn{2}{|l|}{ Total des appels } & \multicolumn{2}{l|}{\begin{tabular}{l}
\multicolumn{2}{l|}{ Appels d'activités } \\
policières
\end{tabular}} & \multicolumn{2}{l|}{$\begin{array}{l}\text { Appels liés à } \\
\text { de la criminalité }\end{array}$} \\
\hline Années & $\begin{array}{c}\text { n. de } \\
\text { mois }\end{array}$ & Moyenne & Écart-type & Moyenne & Écart-type & Moyenne & Écart-type \\
\hline 2006 & 3 & 391,00 & 26,46 & 284,33 & 11,02 & 106,67 & 19,01 \\
\hline 2007 & 12 & 372,33 & 26,75 & 268,00 & 24,69 & 98,75 & 12,21 \\
\hline 2008 & 12 & 366,08 & 37,85 & 257,25 & 30,43 & 108,75 & 13,55 \\
\hline 2009 & 12 & 389,17 & 97,72 & 283,33 & 81,97 & 105,83 & 23,22 \\
\hline Total & 39 & 377,03 & 59,41 & 270,67 & 50,20 & 104,62 & 16,98 \\
\hline
\end{tabular}

8. Nous avons toutefois observé, entre les mois de juin, juillet et août 2009 , une hausse de l'ensemble des appels logés au SSPTR pour les secteurs de patrouille autour du Salon de jeu. Or, vérifications faites auprès des analystes du SSPTR, la hausse est tout simplement attribuable à un changement administratif: un événement annuel vient marquer les statistiques des activités policières dans les secteurs de patrouille situés autour du Salon de jeu, il s'agit de la libération des véhicules entreposés tout au long de l'année à la fourrière municipale (ces véhicules peuvent être retournés à leur propriétaire, revendus aux encan locaux, ou encore, déclarés produit de la criminalité). Or, pendant les années 2006-2007 et 2007-2008, ces activités étaient enregistrées au quartier général du SSPTR et étaient en conséquence exclues de nos analyses; le SSPTR a cependant pris la décision administrative d'enregistrer ces événements à une autre adresse que celle attribuée au quartier général pour la dernière année que nous avons comptabilisé, une adresse cependant située dans les secteurs que nous analysons ici. 
TABLEAU 2

Résultats des analyses de variance sur les moyennes des appels logés par mois à la police pour les années 2007, 2008 et 2009

\begin{tabular}{|c|c|c|c|c|}
\hline Variables et sources & Somme des carrés & Moyenne des carrés & $F(3,35)$ & $p$ \\
\hline Total des appels & & & & \\
\hline Inter-groupes & 4055,724 & 1351,908 & 0,364 & 0,780 \\
\hline Intra-groupes & 130071,250 & 3716,321 & & \\
\hline $\begin{array}{l}\text { Appels d'activités } \\
\text { policières }\end{array}$ & & & & \\
\hline Inter-groupes & 4731,083 & 1577,028 & 0,606 & 0,615 \\
\hline Intra-groupes & 91037,583 & 2601,074 & & \\
\hline $\begin{array}{l}\text { Appels liés à de } \\
\text { la criminalité }\end{array}$ & & & & \\
\hline Inter-groupes & 648,397 & 216,132 & 0,734 & 0,539 \\
\hline Intra-groupes & 10312,833 & 294,652 & & \\
\hline
\end{tabular}

Les chiffres du tableau 2 confirment d'ailleurs l'absence de modification significative, d'un mois à l'autre, à la fois des appels sans criminalité et des appels qui concernent un acte criminel: aucun des indices des analyses de variance n'est significatif?.

Une double question peut maintenant se poser: peut-il y avoir eu, d'une part, déplacement de la criminalité ${ }^{10}$ et, d'autre part, une modification des activités criminelles organisées, des modifications qui n'apparaîtraient peut-être pas distinctement dans les données qui ont été mises à notre disposition par la police locale? C'est afin de trouver réponse à de telles questions que nous avons réuni six représentants $\mathrm{du}$ SSPTR à deux reprises pendant nos trois années de suivi; il s'agissait des mêmes représentants dans les deux occasions.

Pour ce qui est tout d'abord des changements perceptibles par les policiers, qu'il s'agisse de la criminalité ou des habitudes des joueurs, rien de significatif n'est apparu dans la foulée de l'ouverture du Salon de jeu. Très clairement, les adeptes d'ALV qui avaient leurs habitudes

9. Les analyses d'autocorrélation temporelle réalisées ne font pas état non plus d'indices significatifs.

10. Nous avons procédé à l'étude des appels dans d'autres secteurs ciblés du territoire, afin de vérifier qu'il y ait pu y avoir ou non quelque forme de déplacement potentiel de l'activité criminelle ou de l'activité policière; or, et sans aller plus avant compte tenu du format réduit de cet article, là non plus, aucune pointe ou tendance asymptomatique ne ressort de ces analyses. 
dans les bars de la région n'ont en rien modifié ces habitudes à l'ouverture du Salon de jeu:

Un policier superviseur de patrouille, $2^{\mathrm{e}}$ entretien: «Quand on arrive dans les bars le matin, y'a encore du monde qui joue dans leurs p'tits bars; le Ludoplex on dirait c'est plus comme une sortie, les gens y vont plus pour sortir, mais y'en a des babitués sûrement aussi là mais...»

Les raisons évoquées par nos informateurs tiennent en partie aux modalités de contrôle du jeu excessif mises en place par Loto-Québec au Salon de jeu, des modalités qui feraient en sorte que l'utilisation des ALV y serait très différente:

Un policier gestionnaire, $2^{\mathrm{e}}$ entretien: «À ce qu'on en sait, c'est que les gens qui fréquentaient les bars continuent à fréquenter les bars. Pour quelques-uns, c'est une sortie un petit peu plus officielle d'aller au Ludoplex, c'est plus chic, mais la façon de dealer avec le gain, l'bistoire des cartes à puce et non pas d' l'argent, ben ça change toute la dynamique. C'est peut-être pas la même chose exactement.»

Lautre phénomène évoqué par nos informateurs pour expliquer le fait que le Salon de jeu ne semble pas détourner la clientèle fidèle aux bars tient justement à sa faible fréquentation:

Un policier superviseur de patrouille, $1^{\text {er }}$ entretien: "Moi j'aurais un questionnement: (...) mettons que moi, demain matin j'y vais, et c'est la première fois, je suis jamais rentré dans le Ludoplex, et y'a pas un chat, ça a l'air plate [ennuyant], eh bien, $j^{\prime} y$ reviendrais plus icitte... Se pourrait-il que ce soit jamais les mêmes personnes ou presque qui y vont? Les gens se diraient "Câlique, c'est ben plate icitte, je suis venu pour mon année, ça a l'air qu' il n'y a pas de monde, y'a pas personne, je ne reviens plus."”

Cette perception d'une absence de quelque changement que ce soit semble tout aussi vraie pour ce qui est de la criminalité en général que du crime organisé:

Un enquêteur, $1^{\text {er }}$ entretien: "On n'a pas observé quoique ce soit dans les différents établissements licenciés ou bien au Ludoplex pour ce qui est de la présence de crime organisé. À Trois-Rivières, y'a pas de crime organisé, que ce soit asiatique ou autre en lien avec le Ludoplex ou les appareils vidéo. D'autres délits comme la violence conjugale, la fraude, l'extorsion, ces affaires-là... peu pour ne pas dire pas du tout. (...) que ce soit des gens qui volent par effraction ou qui font des fraudes qui a fait ça pour des problématiques de jeu, le dernier je ne mén rappelle même pas. Je ne sais même pas si on n'a eu...»

Un patrouilleur, $2^{\mathrm{e}}$ entretien: "La commission de délits, c'est bien plus relié à la problématique de la drogue que du jeu... Beaucoup plus relié à la problématique de 
la drogue que du jeu, y'a aucune comparaison possible. C'est vraiment les... j'veux dire si tu pognes nos voleurs dans le centre-ville, qui peuvent voler dans les vébicules les cartes de crédit, l'argent, c'est bien plus pour la drogue que pour le jeu.»

Il semble également que le climat de coopération entre les personnes chargées de la sécurité au Salon de jeu et les représentants du service de police local est très sain, ce qui pourrait en partie aussi expliquer l'absence de problèmes significatifs:

Un policier gestionnaire, $2^{\mathrm{e}}$ entretien: "À l'implantation du Salon de jeux, on l'avait mentionné, c'est qu'on avait eu des échanges avec eux autres, on avait eu des présentations. Sur le terrain de façon ponctuelle, si on a des besoins, on rencontre les gens de la sécurité de Loto-Québec, on a une collaboration pleine et entière.»

Les propos tenus en groupe de discussion font globalement état que si des problèmes sociaux se déclaraient ou s'aggravaient à la suite de l'ouverture du Salon de jeu, ce ne serait probablement pas les policiers qui le constateraient:

Un superviseur de patrouille, $1^{\text {er }}$ entretien: "Moi j'ai l'impression que des fois, j'sais pas, ça peut être un travaillant, ça peut être une personne qui est pas criminalisée, qui peut avoir un gros problème de jeu, mais il va peut-être y laisser sa peau ou ses biens mais c'est pas nécessairement quelquiun qui va faire des grands coups, mais des p'tits crimes... Ce qui fait qu'on ne le voit pas comme tel. Moi là, le monsieur qui vient de Pointe-du-Lac qui a peut-être joué son char pis la moitié de l'bypothèque de sa maison... il va aller se faire soigner puis il va vendre sa maison et il va régler ses problèmes, etc. Mais moi je l'aurai pas dans les pattes ce problèmelà. Ici, on ne parle pas de problème de criminalité.»

En résumé, donc, les statistiques tout comme les propos tenus par les informateurs du service de police convergent: l'introduction de cette nouvelle modalité de jeux de hasard et d'argent semble n'avoir affecté ni les données de la criminalité ni celles de l'activité policière. Deux phénomènes expliqueraient, toujours selon les policiers, l'absence d'impact, tant dans les chiffres que dans les perceptions, à savoir les mesures de contrôle mises en place par Loto-Québec d'une part, et l'apparente difficulté de la société d'État à attirer le volume de clientèle espéré, un élément sur lequel nous allons revenir. Ceci étant, nous allons maintenant nous attarder plus spécifiquement sur les tendances quant aux éventuelles fluctuations de demandes d'aide et de référence pour problèmes de jeu pathologique. 
Les demandes d'aide et de référence pour problèmes de jeu pathologique, bilan de trois années de suivi

Dans le cadre de nos travaux à Trois-Rivières, deux sources de données du milieu de la santé et des services sociaux ont pu être exploitées, soit, d'une part, l'évolution des demandes d'aide et de référence pour des problèmes de dépendance au jeu, des données compilées, rappelons-le par le centre de réadaptation en dépendance Domrémy MauricieCentre-du-Québec et, d'autre part, les propos tenus par des informateurs du milieu de la santé et des services sociaux lors de deux séances en groupe de discussion tenues à un an d'intervalle et regroupant les mêmes organismes dans les deux occasions. Examinons tout d'abord les données chronologiques.

\section{F I G URE 1}

Nombre de demandes de référence pour problème de jeu pathologique enregistrées à Domrémy MCQ, de 2006 à 2009

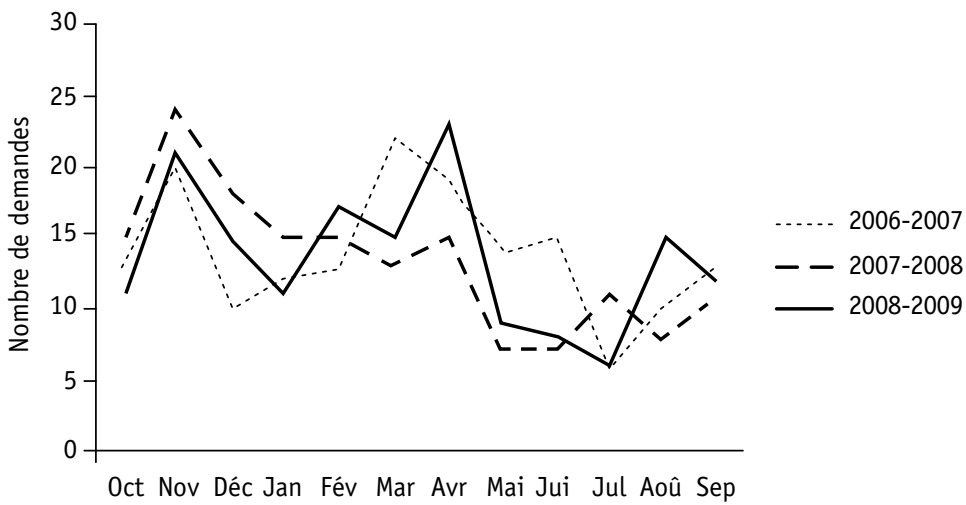

La figure précédente illustre en superposition l'évolution des demandes compilées par l'organisme Domrémy sur trois périodes, soit toute l'année qui précède l'ouverture du Salon de jeu $(2006$ - 2007) et les deux années suivant cet événement. Comme on peut le constater, les demandes vont d'un peu plus de 5 par mois à un peu moins de 25 par mois. La superposition des trois périodes analysées montre à quel point les fluctuations des demandes obéissent essentiellement à une logique saisonnière (une hausse des demandes au mois de novembre et des baisses marquées en juillet, pour ne nommer que ces deux phénomènes) 
et que, surtout, rien ne semble distinguer la période pré-ouverture (2006 - 2007) de la période du début du fonctionnement du Salon de jeu $(2007$ - 2008, et 2008 - 2009).

Mis à part des mouvements essentiellement saisonniers, les données n'apparaissent pas différentes entre les trois différentes périodes. Bien sûr, il s'agit ici de données agrégées pour l'ensemble des onze points de service de la région. Il convient donc maintenant de vérifier de plus près la question des différences potentielles que l'on pourrait remarquer entre, d'une part, la fluctuation des demandes effectuées au point de service de Trois-Rivières et, d'autre part, celle des demandes faites dans les dix autres points de service en région.

TABLEAU 3

Statistiques descriptives des demandes d'aide et de référence adressées à l'organisme Domrémy pour problèmes de jeu pathologiques par mois pour les années 2007, 2008 et 2009

\begin{tabular}{|l|c|c|c|c|c|c|c|}
\hline & & \multicolumn{2}{|l|}{$\begin{array}{l}\text { Demandes pour } \\
\text { l'ensemble des } \\
\text { centres de service } \\
\text { de la Mauricie }\end{array}$} & \multicolumn{2}{l|}{$\begin{array}{l}\text { Demandes pour les } \\
\text { points de service } \\
\text { de Trois-Rivières } \\
\text { seulement }\end{array}$} & \multicolumn{2}{l|}{$\begin{array}{l}\text { Demandes pour les } \\
\text { points de service } \\
\text { de l'extérieur de } \\
\text { Trois-Rivières }\end{array}$} \\
\hline Années & $\begin{array}{l}\text { n. de } \\
\text { mois }\end{array}$ & Moyenne & Écart-type & Moyenne & Écart-type & Moyenne & Écart-type \\
\hline 2007 & 12 & 13,4 & 4,76 & 3,4 & 1,88 & 10,0 & 3,95 \\
\hline 2008 & 12 & 13,5 & 4,87 & 4,1 & 2,02 & 9,4 & 3,58 \\
\hline 2009 & 12 & 13,6 & 5,11 & 3,4 & 2,35 & 10,2 & 3,30 \\
\hline Total & 36 & 13,5 & 4,77 & 3,6 & 2,06 & 9,9 & 3,53 \\
\hline
\end{tabular}

Tout comme dans le cas des séries illustrées en figure 1, les moyennes mensuelles de l'ensemble des demandes demeurent très stables d'une année à l'autre et mis à part une légère différence en 2008, les données découpées selon qu'elles proviennent de Trois-Rivières seulement ou des autres points de service en région demeurent à peu près les mêmes d'une année à l'autre. Cette absence de différence est également confirmée par les analyses de variance dont les résultats sont exposés au tableau 4, les seuils de signification étant tous non significatifs.

Cette absence de lien entre le nombre de demandes et la mise en activité du Salon de jeu est corroborée par les intervenants rencontrés lors des entretiens de groupe. En effet, selon ces intervenants, le Salon de jeu semble avoir maintenu un taux de fréquentation stable depuis son ouverture. 
TAB LEA U 4

Résultats des analyses de variance sur les moyennes des demandes d'aide et de référence adressées à l'organisme Domrémy pour problèmes de jeu pathologiques par mois pour les années 2007, 2008 et 2009

\begin{tabular}{|l|c|c|c|c|}
\hline Variables et sources & Somme des carrés & Moyenne des carrés & $F(2,33)$ & $p$ \\
\hline Total des demandes & & & & \\
\hline Inter-groupes & 0,167 & 0,083 & 0,003 & 0,997 \\
\hline Intra-groupes & 796,833 & 24,146 & & \\
\hline $\begin{array}{l}\text { Demandes à Trois- } \\
\text { Rivières }\end{array}$ & & & & \\
\hline Inter-groupes & 3,556 & 1,778 & 0,405 & 0,670 \\
\hline Intra-groupes & 144,750 & 4,386 & & \\
\hline $\begin{array}{l}\text { Demandes à l'extérieur } \\
\text { de Trois-Rivières }\end{array}$ & & & & \\
\hline Inter-groupes & 3,722 & 1,861 & 0,142 & 0,868 \\
\hline Intra-groupes & 432,583 & 13,109 & & \\
\hline
\end{tabular}

Un intervenant, $2^{\mathrm{e}}$ entretien: "Il n'y a pas nécessairement de hausse, je pense qu'on est dans les... la clientèle qui est habituée d'aller jouer... Oui, on peut voir des nouveaux clients... Ils viennent tester et ils repartent, et des fois on ne les revoit jamais. "

Tout comme les policiers le faisaient également remarquer, la clientèle fréquentant les bars ne semble pas avoir modifié ses habitudes:

Un autre intervenant, $2^{\mathrm{e}}$ entretien: "Ceux qui étaient habitués d'aller dans les bars sont allés faire un tour au Ludoplex, mais ils trouvent que ça ne paye pas, ça fait qu' ils reviennent dans les bars, et là ils ont des places attitrées, et ils disent: "Là, ça paye." "

Sur une note positive, des intervenants s'entendent sur le fait que l'ouverture du Salon de jeu se serait accompagnée d'une augmentation significative des subventions qui leur sont versées:

Un intervenant, $2^{\mathrm{e}}$ entretien: "Depuis deux ans avec $X$ de notre côté, regarde moi j’ai eu $5000 \$$ l'année passée pour faire de la prévention du jeu dans les groupes cibles, Âge d'or, Centres d'action bénévoles, ces choses-là, et cette année on a signé pour 10000 \$ parce que moi, c'est de l'argent tagué [étiqueté] jeu pathologique.»

Ces sommes supplémentaires permettent l'engagement de nouveau personnel et la mise sur pied de nouveaux programmes ou le prolongement des programmes existants: 
Un intervenant, $2^{\mathrm{e}}$ entretien: «ce que ça nous a apporté nous comme changement, c'est des argents supplémentaires, c'est l'ajout d'un poste chez nous qui est dédié à la dépendance (...) c'est le développement d'un programme de prévention, de promotion de première ligne. Donc, c'est une valeur ajoutée d'intervention.»

L'Agence de la santé et de services sociaux Mauricie-Centre-duQuébec dépêche une intervenante appelée à jouer le rôle d'ambassadrice du jeu responsable au Salon de jeu de Trois-Rivières. Or, dans ce cas précis, l'intervention et la dispense de conseils sur les lieux mêmes où sont les ALV semblent se dérouler dans un contexte plus difficile:

Une intervenante, $1^{\text {er }}$ entretien: "Mon nom n'est pas affiché, (...) il faudrait arriver à pouvoir développer quelque chose en ce sens-là pour que les gens sachent, oui vraiment, c'est une intervenante en qui on peut avoir confiance, en qui on pourrait avoir un service confidentiel, mais je n'ai pas de local où les gens peuvent venir cogner.»

Cette intervenante déplore le fait que l'intervention et la prévention qu'elle fait au Salon de jeu doivent se dérouler selon les termes de LotoQuébec:

Une intervenante, $1^{\text {er }}$ entretien: «On n'est pas dans un aspect de collaboration, de partenariat avec eux autres, on est vraiment dans un aspect reddition de compte. On a consciemment accepté de travailler comme ça parce que c'était l'ouverture qu'on avait et on s'est dit à partir de cette ouverture-là, on créera autre chose...»

Ceci étant, les propos tenus par les intervenants lors du deuxième entretien de groupe un an plus tard démontrent une amélioration du climat de collaboration entre eux et les autorités locales de Loto-Québec; en effet, si la première rencontre que nous avons eue faisait ressortir certaines difficultés à cet égard, la seconde rencontre a permis d'établir que les choses s'étaient améliorées.

Il reste toutefois que globalement, et au vu de nos indicateurs, aucune des craintes exprimées par les représentants de la santé publique avant l'ouverture du Salon de jeu ne s'est matérialisée: aucune variation significative n'est remarquée dans l'évolution de demandes de référence pour jeu pathologique.

\section{Conclusion}

À ce stade-ci de nos travaux et pour ce qui est des données touchant la criminalité et les activités policières, nos analyses confirment ce que la littérature a jusqu'ici assez clairement établi: tant ce qui a été observé 
autour des casinos ailleurs dans le monde que pour le cas du Salon de jeu à Trois-Rivières, ces modalités d'offre de jeux de hasard et d'argent semblent n'avoir aucun impact mesurable sur la criminalité et la délinquance, peu importe les mesures prises. Ceci dit, il n'en demeure pas moins que le Salon de jeu de Trois-Rivières propose des jeux de hasard et d'argent qui se font dans des conditions quasi optimales de contrôle. En d'autres termes, la gestion que fait la société Loto-Québec des installations du Salon de jeu à Trois-Rivières correspond à ce que la recherche sur les modalités sûres d'offre de jeux de hasard et d'argent a clairement démontré: une bonne collaboration avec les autorités policières locales, des dispositifs de sécurité interne visibles et efficaces et un monitorage serré de l'offre de jeux constituent les meilleures méthodes pour éviter d'éventuels débordements qui auraient des répercussions mesurables, ne serait-ce que sur l'activité policière (Wynne et Shaffer, 2003 ; Blaszczynski et al., 2004).

Dans le cas de notre seul indicateur quantitatif de santé et de services sociaux, cependant, les choses sont moins claires. Elles le sont, bien sûr, pour ce qui est du constat de l'absence de relation entre l'ouverture $\mathrm{du}$ Salon de jeu et les fluctuations du nombre de demandes d'aide et de références pour jeu pathologique, mais elles le sont moins pour ce qui est des interprétations que l'on peut faire de ce constat. Le problème est qu'ici, la littérature va dans le sens contraire de ce que nous observons à Trois-Rivières: les données cumulées, tant au Canada (Afifi et al., 2010), qu'en Nouvelle-Zélande (Pearce et al., 2007) qu'au Québec (Bélanger et al., 2003) montrent que l'augmentation de l'offre de jeux de hasard et d'argent d'une part, et la facilité d'accès à des ALV, d'autre part, constituent des facteurs d'accroissement du nombre de personnes aux prises avec une habitude de jeu problématique. Ce constat était résumé ainsi par Korn, Gibbins et Azmier (2003):

These findings support the general conclusion that gambling expansion has been associated with increases in problem and pathological gambling. (Korn et al., 2003: 240)

Ce sont de tels constats, d'ailleurs, qui alimentaient les craintes exprimées par les directeurs de la santé publique du Québec dans leur avis sur l'implantation des salons de jeux dans la province:

Les données disponibles ne permettent pas d'affirmer que le développement de salons de jeux soit une mesure qui restreindra au plan régional l'accessibilité aux ALV, notamment parce qu'il se fera dans des zones densément 
peuplées. Les nombreux facteurs de vulnérabilité présents dans plusieurs de ces secteurs accentuent les impacts négatifs potentiels. (...) Finalement, les mesures préventives proposées sont minimales et imputent le fardeau de la responsabilité essentiellement au joueur. (Houle et Paradis, 2007: 8)

Or, nos analyses des données quantitatives et celles des propos des intervenants du milieu de la santé et des services sociaux sont cohérentes sur ce point: l'ouverture du Salon de jeu ne s'est pas accompagnée d'une modification du nombre de demandes d'aide. De plus, compte tenu du fait que les données mises à notre disposition permettent de jauger de la possibilité de déplacements (c.-à-d. lorsqu'une personne de Trois-Rivières qui éprouverait le besoin de se faire conseiller irait le faire à l'extérieur de la ville, dans l'un ou l'autre des 10 autres points de service régionaux), rien n'indique qu'un tel phénomène ait pu jouer. Il convient alors de se poser la question de savoir quelle est la nature et l'ampleur de la relation entre les demandes d'aide, d'une part, et le nombre de personnes qui éprouvent un problème de jeu pathologique, d'autre part. Il se pourrait, à titre d'exemple, qu'entre l'apparition d'un problème de jeu chez un joueur et le moment où il décide d'aller demander de l'aide, il s'écoule un laps de temps qui soit au-delà de la période de temps que nous avons été en mesure d'analyser. L'idée avancée alors par les directeurs de la santé publique, à l'effet que «les problèmes de jeu sont attribuables à l'accessibilité des jeux en conjonction avec leur dangerosité» (Houle et Paradis, 2007: 11), ne pourrait se vérifier qu'en fonction d'un monitorage à plus long terme des demandes d'aide et de référence. Chose certaine, cette crainte et plusieurs autres avancées par les directeurs de la santé publique ne se sont pas matérialisées au cours des deux années qui ont suivi l'ouverture du Salon de jeu.

Pour en revenir à une perspective que nous avons survolée en introduction à cet article, soit celle de considérer l'offre étatique de jeux de hasard et d'argent sous la lunette de la santé publique, on pourra avancer que les modalités de contrôle prônées par cette perspective sur le plan du fonctionnement des ALV ont toutes été respectées au Salon de jeu, soit le temps (c.-à-d. un rappel affiché sur les ALV du Salon de jeu de même que sur tous les modèles les plus récents partout au Québec informe le joueur du temps qu'il passe à jouer), l'argent (au Salon de jeu, on ne met ni ne retire d'argent en espèce des ALV) et le contrôle du joueur (cette idée qui se forge peu à peu chez le joueur pathologique qu'il possède une part de contrôle sur la machine est démentie par des 
bandes vidéo qui passent sur son écran) (Houle et paradis, 2007 : 18). Cependant, la position plus générale de la perspective de la santé publique proposée, notamment par Korn et ses collègues, introduit un autre grand principe que l'on ne retrouve pas vraiment mentionné dans le rapport des directeurs de la santé publique du Québec de 2007 : c'est celui voulant qu'une offre publique censée de jeux de hasard et d'argent repose sur un équilibre entre, d'une part, la gestion des risques associés, tant sur le plan individuel que collectif et, d'autre part, la démonstration de retombées économiques positives pour l'ensemble de la communauté, ces retombées constituant alors une plus-value collective. Or, c'est probablement là plus qu'ailleurs qu'un déséquilibre significatif affecte le projet de Trois-Rivières: si l'on peut avancer que les coûts sociaux potentiels liés à l'implantation du Salon de jeu sont demeurés extrêmement limités, en revanche, les retombées économiques l'ont été probablement tout autant.

Les chiffres de fréquentation compilés par les gestionnaires responsables du Salon de jeu sont bien en deçà de ce que Loto-Québec espérait atteindre au moment de l'ouverture ${ }^{11}$; si la société d'État comptait en effet attirer entre 1000 et 1200 joueurs par jour, un article publié dans un quotidien local (Le Nouvelliste, 13 février 2008) faisait état d'une moyenne située un peu en dessous de la moitié de ce chiffre. L'affaire est telle, d'ailleurs, que les marges de profit espérées par les gens de l'industrie des courses de chevaux sont à ce point basses que l'une des principales compagnies qui les représente, Attractions hippiques du Québec, a dû se mettre sous la protection de la Loi sur les créanciers dès juin 2008 (Le Nouvelliste, 27 juin 2008).

Pour en finir avec la polémique qui a constitué le moteur premier de nos travaux empiriques et toujours dans le cadre restreint en temps et en nature des indicateurs dont nous avons disposés, nous devons reconnaître qu'à l'heure actuelle, ni Loto-Québec et les supporteurs du Salon de jeu pour ce qui est des profits espérés, ni les directeurs de santé

11. Un représentant de Loto-Québec nous a fourni les chiffres exacts de fréquentation du Salon de jeu de Trois-Rivières pour l'ensemble de l'année 2009; l'installation a suscitée 105880 visites (notre propre estimation, à partir des données d'un sondage que nous avons commandé, fait plutôt état de 67350 visites; il est plus que probable que les 38530 visites manquantes du chiffre réel correspondent à celles effectuées par les visiteurs de la région ou d'ailleurs qui, évidemment, ne sont pas comptabilisées dans notre sondage). Or, même là, nous sommes encore assez loin du niveau d'achalandage annoncé au moment de l'ouverture, soit 30800 visites par mois. Avec une moyenne mensuelle de 8823 visites, ce n'est que $29 \%$ de l'achalandage prévu. 
publique pour ce qui est des effets délétères de cette initiative sur les indices de santé publique, ne semblent avoir eu raison. Sur un plan un peu plus théorique, finalement, nous avons été en mesure de montrer que l'idée de considérer l'offre de jeux de hasard et d'argent comme une donnée de santé publique semble faire sens et que le peu d'attrait exprimé par les populations locales pour le Salon de jeu a fort probablement constitué l'une des principales raisons du peu de problèmes constatés, mais aussi du peu de retombées économiques significatives observées.

\section{Références}

Afifi T., Cox, B., Martens, P., Sareen, J., \& Enns, M. (2010). The relation between types and frequency of gambling activities and problem gambling among women in Canada. The Canadian Journal of Psychiatry, 55 (1), 21-28.

Alain, M., Brunelle, N., Dessureault, D., \& Crête, C. (2009). When something goes wrong: Loto-Quebec's strategy to concentrate the offer of slot machines in mini-casinos. Worldwide Hospitality and Tourism Themes, 1 (4), 379-385.

Bélanger, Y., Boisvert, Y., \& Papineau. É. (2003). Les responsabilités de l'État en matière de jeu patbologique: la gestion des $A L V$. Montréal: INSPQ-ENAP.

Blaszczynski, A., Ladouceur, R., \& Shaffer, H. (2004). A science-based framework for responsible gambling: The Reno model. Journal of Gambling Studies, 20 (3), 301-317.

Chang, S. (1996). Impact of casinos on crime: The case of Biloxi, Mississippi. Journal of Criminal Justice, 24 (5), 431-436.

Chevalier, S., Hamel, D., Ladouceur, R., Jacques, C., Allard, D., \& Sévigny, S. (2004). Comportements de jeu et jeu pathologique selon le type de jeu au Québec en 2002. Institut National de Santé publique Québec et Université Laval.

Chevalier, S., \& Papineau, É. (2007). Analyse des effets sur la santé publique de l'aménagement des salons de jeux et des hippodromes. Québec, QC: Directeurs régionaux de santé publique.

Cohen, L. E., \& Felson, M. (1979). Social change and crime rate trends: A routine activity approach. American Sociological Review, 44, 588-608.

Felson, M. (1998). Crime and everyday life. Thousand Oaks: Pine Forge Press.

Gottfredson, M., \& Hirschi, T. (1991). A general theory of crime. Stanford: Stanford University Press.

Grinols, E. L. (2000). Casino gambling causes crime. Policy Forum, 13 (2), 1-4.

Houle, V., \& Paradis, L. (2007). Avis de santé publique sur l'implantation des salons de jeux au Québec. Québec: Les Directeurs régionaux de santé publique.

Korn, D. A. (2000). Expansion of gambling in Canada: Implications for health and social policy. CMAJ, 163, 61-64.

Korn, D., Gibbins, R., \& Azmier, J. (2003). Framing public policy towards a public health paradigm for gambling. Journal of Gambling Studies, 19 (2), 235-256. 
Korn, D., \& Shaffer, H. (1999). Gambling and the health of the public: Adopting a public health perspective. Journal of Gambling Studies, 15 (4), 289-365.

Korn, D. A., \& Skinner, H. A. (2000). Gambling expansion in Canada: An emerging public health issue. CPHA Health Digest, XXIV, 10.

Ladouceur, R., Blaszczynski, A., \& Pelletier, A. (2004). Why adolescent problem gamblers do not seek treatment. Journal of Child and Adolescent Substance Abuse, 13 (4), 1-12.

Leblond, J. (2007). Dangerosité des appareils de jeux et mesures de protection. Document d'analyse remis au directeur de santé publique de la Capitale-Nationale, Québec, QC.

Loto-Québec (2007). Reconfiguration du réseau des appareils de loterie vidéo : objectif 2007 Loto-Québec poursuit son initiative en vue de réduire l'offre de jeu au Québec. Consulté le 18 janvier 2007, http://www.loto-quebec.com/corpo/ nav/salle-de-presse/communiques.

Mays, G. L., Casillas, C., \& Maupin, J. R. (2007). The impact of Indian gaming on crime in New Mexico: A research note. The Social Science Journal, 44, 375-381.

Mun, W. P. (2003). Calculating risk-taking: The governance of casino gambling in Ontario. Dissertation Abstract International, 63 (12), 4484-A.

Pearce, J., Mason, K., Hiscock, R., \& Day, P. (2008). A national study of neighbourhood access to gambling opportunities and individual gambling behaviour. Journal of Epidemiological Community bealth, 62, 862-868.

Piscitelli, F., \& Albanese, J. S. (2000). Do casinos attract criminal? A study at the Canadian-U.S. border. Journal of Contemporary Criminal Justice, 16 (4), 445-456.

Powel, H. C. (2004). The social impact of riverboat casino gambling: Using objective local indicators to measure changes in the quality of life in a gambling community. Dissertation Abstract International, 64 (10), 3849-A.

Shaffer, H. J., \& Korn, D. A. (2002). Gambling and related mental disorders: A public health analysis. Annual Review of Public Health, 23, 171-212.

Shaffer, H., Labrie, R., \& LaPlante, D. (2004). Laying the foundation for quantifying regional exposure to social phenomena: Considering the case of legalized gambling as a public health toxin. Psychology of Addictive Behavior, $18(1), 40-48$.

Slutske, W. S., Blaszczynski, A., \& Martin, N. G. (2009). Sex differences in the rates of recovery, treatment-seeking, and natural recovery in pathological gambling: Results from an Australian community-based twin survey. Twin Research and HumanGenetics, 12 (5), 425-432.

Smith, G. S., Wynne, H., \& Hartnagel, T. (2003). Examining police records to assess gambling impacts: A study of gambling-related crime in the city of Edmonton. Edmonton: The Alberta Gaming Research Institute.

Wilbon, A. M. (2005). Casinos and crime: A sociological investigation into the relationship between gambling and crime rates in Louisiana. Conference Paper, Southern Sociological Society, Charlotte, North Carolina.

Wilson, J. M. (2001). Riverboat gambling and crime in Indiana: An empirical investigation. Crime and Delinquency, 47 (4), 610-640. 
Wynne H., \& Shaffer, H. (2003). The socioeconomic impact of gambling: The whistler symposium. Journal of Gambling Studies. 19 (2), 111-121.

ABSTRACT - This article presents the first results emerging from a three-year follow-up of the social impacts of a new gaming facility opened and operated by Loto-Québec in the middle-size city of Trois-Rivieres and offering slot-machines, virtual poker tables and a virtual roulette. Two main data sets were analysed in order to provide a general portrait of the post-implantation evolution: (1) longitudinal data from the city's police records, (2) longitudinal data of requests addressed for gambling-related problems at public health services. If, on the one hand and in accordance with the literature, the police data seems to show no truly measurable impacts, on the other hand and contrary to the literature, no true fluctuations of requests for help have been observed. We hereby propose that the main explanation for these phenomena lies in the absence of any real popular success for the new installation during the time we were collecting the data.

KEYWORDS - Gambling, induced crime, induced police activity, legal casinos, pathological gambling, public health

RESUMEN - Este articulo presenta los resultados del seguimiento de los efectos macrosociológicos relacionados con la instauración de una nueva oferta de juegos de azar y de dinero presentados por Loto-Québec en un contexto suburbano: el Salón de Juegos de Trois-Rivières, un espacio de entretenimiento que reagrupa bajo un mismo techo aparatos de video lotería, mesas de Póquer y una ruleta virtual. Varias colectas de información sirvieron para materializar un balance de los efectos, por un lado un seguimiento cronológico de los llamados dirigidos al servicio de policía de la ciudad y, por otro lado, un seguimiento de los pedidos de ayuda y de referencia por los problemas relacionados al juego patológico, pedidos actualizados por el organismo Domrémy MCQ. El análisis de los datos policiales es concordante a lo que revela la literatura, es decir, que no existe relación alguna entre la introducción de una nueva oferta legalizada de juegos de azar y de dinero y la criminalidad. No obstante, la ausencia de relación entre esta nueva oferta y el número de pedidos de ayuda, toma una dirección contraria a lo reflejado en la literatura. Nuestra propuesta consiste en que la causa principal que explica estos fenómenos, reside en el magro éxito que tuvo la sala de juegos durante el periodo en el que se desarrollo nuestro trabajo.

PALABRAS CLAVE - Juegos de azar y de dinero, criminalidad inducida, actividad policial inducida, casinos légales, juego patólogico, salud pública. 